\title{
Bio-Based Building Materials - How to Unravel the Role of Material Characteristics on Fungal Susceptibility?
}

\author{
Liselotte C. De Ligne ${ }^{1,2}$, Jan B.B. Van den Bulcke ${ }^{1}$, Jan M. Baetens ${ }^{2}$, Bernard De Baets ${ }^{2}$ \\ and Joris C.M. Van Acker ${ }^{1}$ \\ ${ }^{1}$ Ghent University (UGent), Laboratory of Wood Technology (UGent-Woodlab), Coupure \\ links 653, 9000 Ghent, Belgium, liselotte.deligne@ugent.be \\ ${ }^{2}$ Ghent University (UGent), Research Unit Knowledge-based Systems (KERMIT), Coupure \\ links 653, 9000 Ghent, Belgium, liselotte.deligne@ugent.be
}

\begin{abstract}
Bio-based materials are gaining importance in the building industry, as the focus on sustainability and life-cycle-assessment has increased substantially over the last decade. Wood and wood-engineered products as well as insulation materials made from cellulose, wood, flax, hemp, etc. are increasingly used. These materials are made from renewable resources and with considerably lower energy consumption than various other building materials, such as insulation polymers, steel and concrete. As steel can corrode and concrete can rot, so can bio-based building materials degrade over time when exposed to those conditions that favour decay. Since fungi cause not only aesthetical degradation, but can also severely compromise the structural integrity of a building component this is critical for any service life approach. Consequently, a proper understanding of the fungal susceptibility of bio-based materials is needed, both for optimal application of bio-based materials as for the design of new materials. Based on a combination of tests we try to unravel the role of the material's chemical components, structure and moisture dynamics on its fungal susceptibility, as well as the interaction between those material characteristics. In a first test set-up, the 'paste test', the material's structure is removed and fungal growth is assessed over time in $2 D$, with only the material's chemical components playing a role. In the second test set-up, the 'X-ray CT test', fungal development is assessed nondestructively in $3 D$ with X-ray CT, giving an indication of moisture production and distribution over time, in relation to the material's structure. By comparing the results, we have a better idea of how much each material characteristic influences fungal susceptibility. This knowledge can then be used for optimising fungal testing of bio-based materials, ensuring optimal application and providing the building industry with the confidence they need to pave the way to a more sustainable future.
\end{abstract}

Keywords: Bio-Based Building Materials, Material Characteristics, Fungal Degradation, X-Ray CT.

\section{Introduction}

The construction sector has a considerable impact on the environment as it consumes large quantities of energy, water and raw materials (Melchert, 2007). In Europe, around 65\% of all aggregates (sand, gravel and crushed rock) and approximately $20 \%$ of all metals are used in this sector (Herczeg et al., 2014). There is, however, a clear trend towards sustainable building projects, both in the residential and industrial construction sector (Jones and Brischke, 2017). Bio-based materials, in casu wood, wood-engineered products and insulation materials made from cellulose, wood, flax, hemp, etc. are key in the sustainable building context. These materials originate from renewable resources and hence have several advantages. They constitute a significant carbon sink when managed sustainably and are produced by consuming considerable less energy than aluminium, steel and concrete (Sathre and O'Connor, 2008; Jones 
and Brischke, 2017). As they are often biodegradable, they are easy to process at the end of their service life (Ganotopoulou, 2014). The latter characteristic is, however, most unwanted while in service. When an organic material is exposed to favourable moisture and temperature conditions as well as to degrading organisms, its functional and aesthetic service life can decrease. Fungi can also cause structural damage and lead to degradation of building materials (Bertron, 2014; Dedesko and Siegel, 2015). It is, for example, well known that most wood products used in outdoor environments must be protected against deterioration by decay fungi (Goodell et al., 2003).

It is widely agreed that the proliferation and growth of fungi on building materials are controlled by the presence of nutrients and sufficiently available water. Moisture problems in buildings can for instance occur when building materials get moist during the construction phase, due to infiltration of rain water or because of condensation (Adan and Samson, 2011). When the environment provides a high relative humidity, porous materials can become sources of water for microorganisms (Hoang et al, 2010; Verdier et al., 2014). Next to spatial structure and the closely related moisture dynamics (Verdier et al., 2014), the chemical composition of a material plays an important role in fungal susceptibility as well. For example, there are several wood species of which the heartwood contains natural fungicidal extractives (Jebrane, 2014; Goode11, 2003). Other less durable materials are either modified or treated with protection products to increase their durability. For instance, solid wood and fibre products are subjected to heat treatment, hydrophobation with oils or resins and chemical modification such as acetylation and furfurylation to increase durability (Homan and Jorissen, 2004).

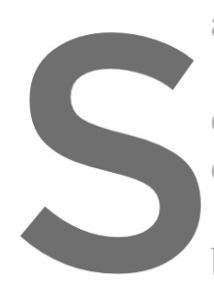
There is little detailed dat except for solid wood determining the natural 15083-1 test method (Cr basidiomycetes is assess subject wood samples $\left(5 \times 2.5 \times 1.5 \mathrm{~cm}^{3}\right)$ for 16 weeks to pure cultures of white rot and brown

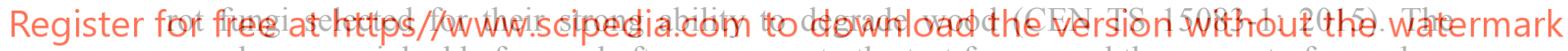
samples are weighed before and after exposure to the test fungus and the amount of mass loss due to degradation is calculated. These standards for the assessment of the efficacy of wood preservatives and of the inherent resistance of wood species are generally regarded to be sufficient and adequate (Kutnik, 2013). However, these standards are typically inadequate for the qualification of new wood products, whose durability is not enhanced with biocides but by new technologies, such as chemically modified wood (acetylation, furfurylation, etc.), thermally treated wood, glue-laminated wood, wood-based panels and wood treated with water repellents (Candelier et al., 2016; Jones and Brischke, 2017; Kutnik et al., 2014; Ringman et al., 2014; Ormondroyd et al., 2015). For example, laboratory tests are performed using conditions optimal for the test fungus. A growth medium is used and serves two purposes: one being nutritional support for the fungus, the other to maintain a high enough moisture content for fungal activity. If the wood does not reach the required moisture content, the test may be declared invalid according to the strict interpretation of the standard (CEN EN 113: 1996, CEN TS 15083-1: 2015). However, thermal and chemical modifications change the wood-water interactions in such a way that the resulting equilibrium moisture content (EMC) of modified woods is lower than the equivalent non-modified wood (Candelier et al., 2016; Mai et al., 2010; Ormondroyd et al., 2015; Ringman et al., 2014). The modified wood samples might not moisten 
fast enough during the 16 weeks testing period for the fungus to reach significant degradation. It does not mean, however, that these materials cannot eventually become wet enough for degradation. Kamden et al. (2002), confirmed that after a period of six weeks exposure, the modified wood samples did become wet and noted moisture contents varying between $72 \%$ and $156 \%$. We therefore need to contemplate whether the set-up of the standard needs to be adapted, for instance by prolonging the test duration or increasing the initial moisture content of the modified wood, or whether fungal degradation of modified wood needs to be observed under the same humidity conditions as for the non-modified wood (Ormondroyd et al.,2015). This example illustrates the importance of understanding certain material characteristics and their influence on fungal resistance as well as the test methodology, in order to properly interpret experimental data derived from standard tests. Consequently, further research is needed on the intricate material-fungal relationship to gain the necessary insights for adequate adaptation of the standard tests, both for optimal application of bio-based materials as for the design of new materials. This is especially pressing given the lack of experience with these new materials compared to the traditional building practice.

In this paper, we propose two tests to unravel the role of the material's chemical components, structure and moisture dynamics on its fungal susceptibility, as well as the interaction between those material characteristics. In a first test set-up, the 'paste test', the material structure is removed and fungal growth is assessed over time in 2D, with only the material's chemical components playing a role. In the second test set-up, the 'X-ray CT test', fungal development is assessed non-destructively in $3 \mathrm{D}$ with $\mathrm{X}$-ray CT, giving an indication of moisture production and distribution over tin have a better idea of how

\section{Assessing the Role}
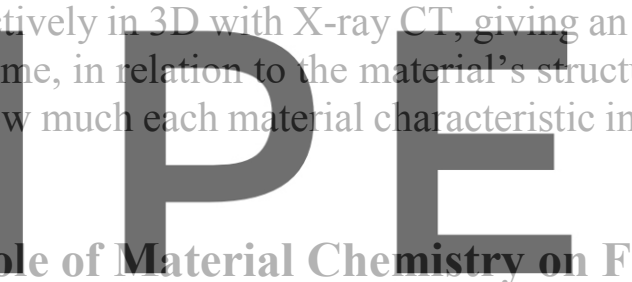

In the paste test, a bio-based material is grinded to a fine powder with a particle size smaller

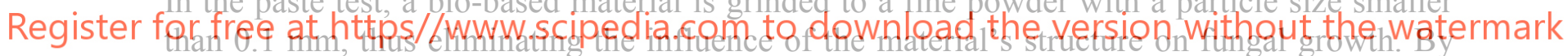

mixing the powder with agar and water, we ensure that the powder is water saturated, thereby aiso limiting the influence of hydrophobic components. The resuiting paste is inoculated with a wood degrading fungus and mycelial growth is assessed over time with a flatbed scanner. The paste test thus gives an indication of how the overall chemistry, consisting of the nutritional value and the presence of toxic components, influences the fungal susceptibility of a bio-based material.

Figure 1 shows the mycelial development of Coniophora puteana, a common wood-rotting basidiomycete, on four bio-based materials. A material with a low natural durability, such as untreated beech wood, does not inhibit mycelial growth (Fig. 1 a). In contrast, a wood species containing toxic components typically limits fungal growth, as is the case for the heartwood of European cherry (Fig. 1 b). The paste test provides additional insights when combined with other tests, for instance assessing the degradation of the material with the structure still intact or of the material with its toxic components removed. In that way, we can find out the influence of the chemistry, and to what extent the durability is due to the material's density or hygroscopic properties. An interesting example is thermally modified wood and derived products, which have been chemically changed because of the wood being heated at high temperatures (Homan 
and Jorissen, 2004). Thermal modification reduces the amount of hydroxyl groups in the wood cellulose and hemicellulose (Mazela et al., 2004). The wood-water interactions of thermally modified wood therefore change in such a way that the resulting EMC is lower than that of the equivalent non-modified wood (Candelier et al., 2016; Mai et al., 2010; Ormondroyd et al., 2015; Ringman et al., 2014). Mazela et al. (2004) determined the fungal resistance of blocks of Scots pine sapwood (Pinus sylvestris L.) subjected to different thermal and hydrothermal treatments. They found that wood blocks exposed to thermal treatments of $190^{\circ} \mathrm{C}$ and $220^{\circ} \mathrm{C}$ were resistant to the test fungi. They hypothesized that besides the thermal effects on wood hygroscopicity, thermal treatment also generates toxins that could affect fungal growth. With the paste test, we can easily verify whether the amount of toxins that are possibly formed, such as furfural or trace quantities of lignin phenol derivates (Mazela et al., 2004), are sufficiently present to inhibit or slow down fungal growth. Figure 1 shows an example of the paste test performed on non-modified (c) and thermally modified (d) three-layer-plywood of spruce. In this case, we can state that the applied modification process did not generate meaningful amounts of toxins, as the mycelial development is not inhibited. The fungal resistance of this particular product therefore stems from its acquired hygroscopic properties.

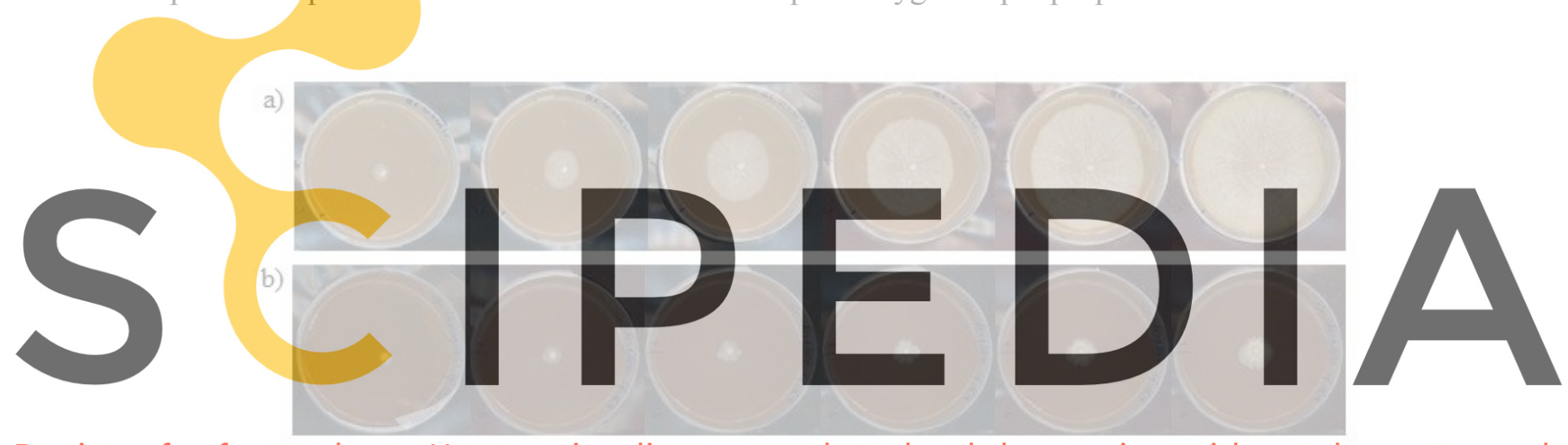

Register for free at https//www.scipedia.com to download the version without the watermark
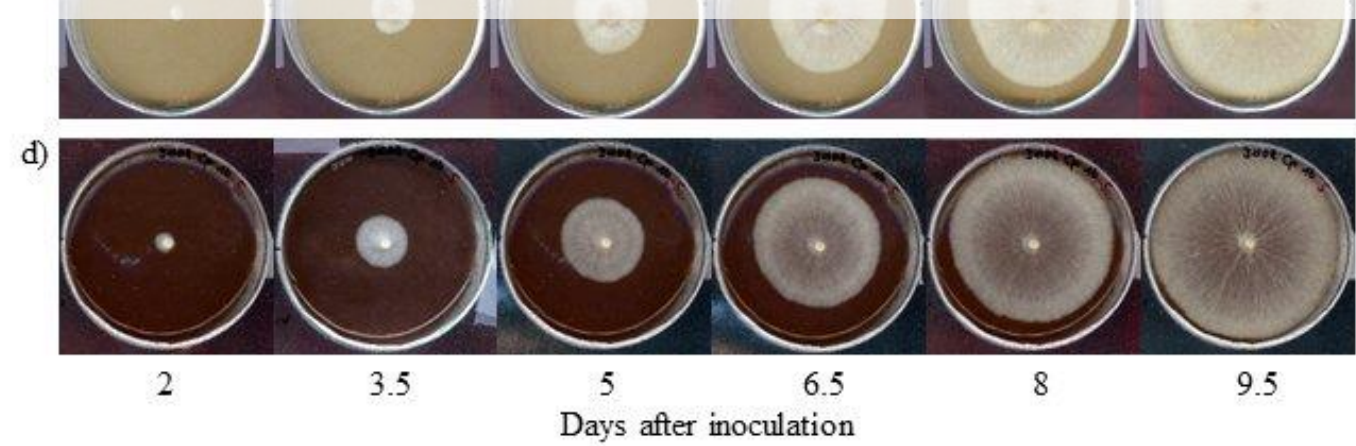

Figure 1. C. puteana growing on a paste of a) beech heartwood b) heartwood of European cherry c) three-layerplywood of spruce and d) thermally modified three-layer-plywood of spruce. 


\section{Assessing the Role of the Material's Structure and Moisture Dynamics on Fungal Susceptibility}

Degradation of (treated) solid wood is usually assessed by measuring the mass loss caused by decay fungi after sixteen weeks (CEN EN 113: 1996, CEN TS 15083-1: 2015). This is an excellent method for testing the durability of a certain wood species or the efficacy of a preservation product, but does not offer much information on what is going on inside the wood structure during degradation. To gain more insight into how the material's structure and moisture properties affect the degradation process, we propose X-ray CT to provide additional information. X-ray CT scanning is a technology that uses X-ray projections to produce tomographic images (slices) of specific areas of a scanned object, allowing the user to inspect the inside of the scanned object in a non-destructive way $(\mathrm{Li}, 2016)$. Due to the different attenuation coefficients between material and air, X-ray CT scanning can reveal the internal spatial structure and density distribution of a material (Bergsten et al., 2001; Chen et al., 2010; Freyburger et al., 2009). It therefore enables us to obtain localized information inside wood during degradation, without disrupting the degradation process. The set-up we designed is able to automatically scan a stack of Petri dishes, one after the other, allowing extensive testing of the degradation process (Fig. 2).
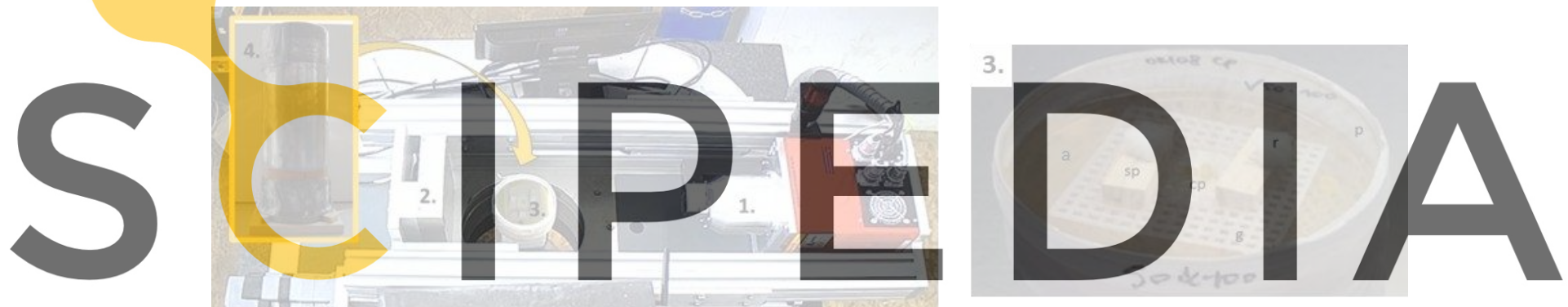

Register for free at https//www.scipedia.com to download the version without the watermark

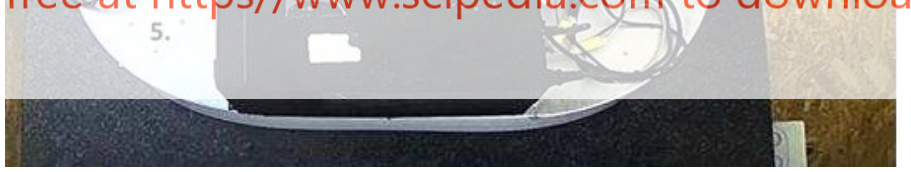

Figure 2: X-ray CT set-up. 1) X-ray source 2) Detector 3) Stack of Petri dishes 4) PVC tube with lead cladding 5) Rotation table. $\mathrm{a}=$ agar medium, $\mathrm{cp}=$ C. puteana, $\mathrm{p}=$ Petri dish, $\mathrm{g}=$ plastic grid, $\mathrm{r}=$ reference material, $\mathrm{sp}=\mathrm{Scots}$ pine

A first test was performed on mini-blocks of Scots pine sapwood under degradation by $C$. puteana, scanned at weekly intervals with X-ray micro CT (De Ligne et al., 2019). We were able to assess the weekly change in density for each wood block. The results are not straightforward to interpret as a consequence of confounding effects, as several factors affect the assessed density: moisture uptake from the agar medium and moisture production by the fungus increase the average density profile, while water evaporation and mass loss due to degradation decrease it. However, we were able to link increasing density to increasing moisture content, which in turn is linked to increasing fungal activity, as fungi metabolise sugars into carbon dioxide and water (Schmidt, 2006). To assess how the material's structure and density influence the fungal degradation process, we are currently performing a test on 
mini-blocks $\left(0.5 \times 1 \times 3 \mathrm{~cm}^{3}\right)$ of four wood species with differing densities: beech (Fagus sylvatica L.) (a), gaboon (Aucoumea klaineana Pierre) (b), Scots pine sapwood (Pinus sylvestris L.) (c) and Norway spruce (Picea abies (L.) Karst) (d). Figure 3 gives a snapshot of the degradation process over time. Darker pixels indicate regions with low density, such as air. White pixels show regions with high density. The white stripes in beech (a), Scots pine sapwood (c) and spruce (d) indicate the latewood zones of the growth rings, which are denser than the earlywood. At the start of the experiment (week 0), no degradation had taken place. White blotches start appearing as degradation progresses over time. These regions with high density are an indication of liquid water production by the fungus, and demonstrate an increasing fungal activity. For Scots pine (c) and spruce (d), degradation is clearly starting from the sides of the mini-blocks (week 1) and progressing towards the center of the mini-blocks (week 4 to 8 ). This is to be expected, since the mini-blocks were cut in such a way that the transverse plane is one the side, providing ample tracheid openings through which hyphae and water can enter the wood.

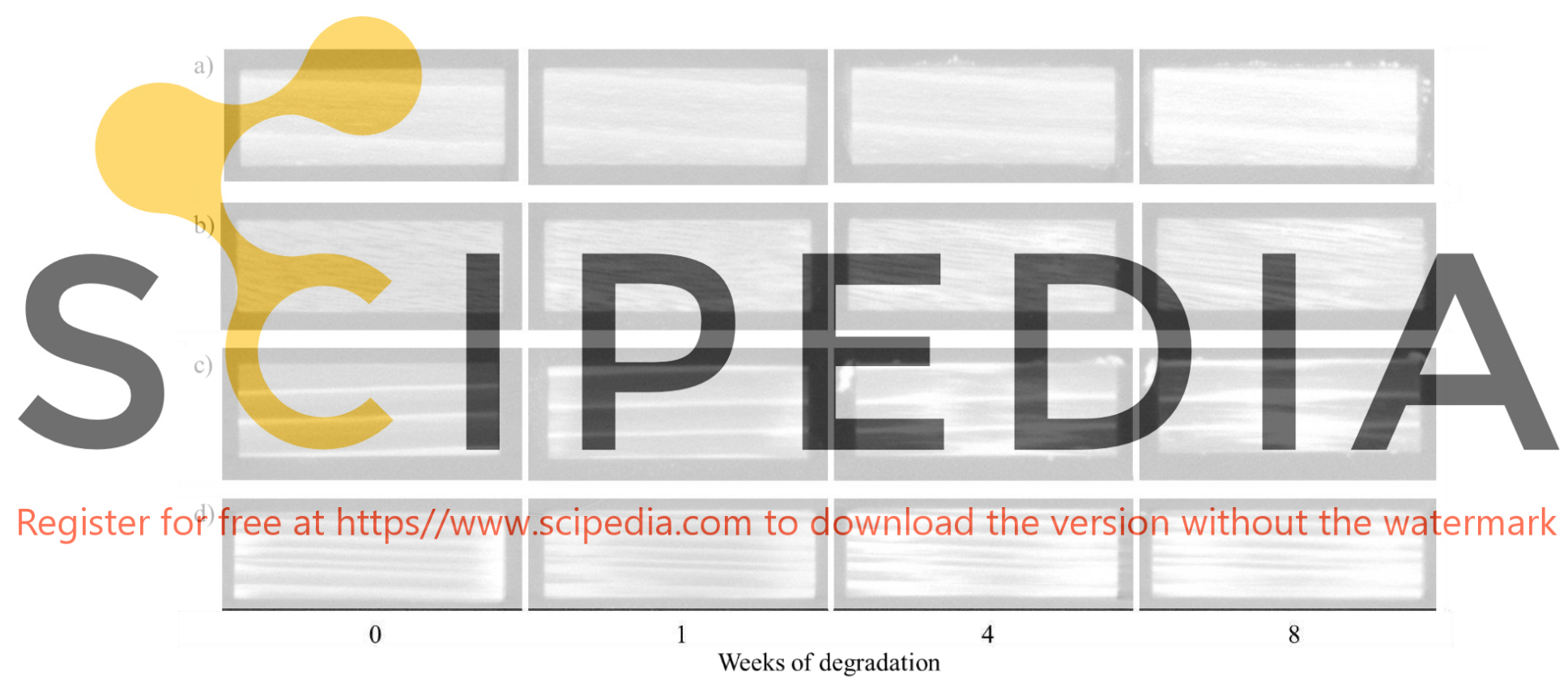

Figure 3. CT slices showing the fungal degradation of beech (Fagus sylvatica L.) (a), gaboon (Aucoumea klaineana Pierre) (b), Scots pine sapwood (Pinus sylvestris L.) (c) and Norway spruce (Picea abies (L.) Karst) (d) mini-blocks over time by $C$. puteana. The whiter the pixels, the higher the density.

\section{Conclusions and Future Perspectives}

- In the paste test, the influence of the material's structure and hygroscopic components on fungal growth is eliminated. It thus provides insight into the role of only the material chemistry on the overall fungal susceptibility of a material. It is currently being applied to a wide range of bio-based materials and will provide useful insights, especially in combination with other test methods.

- The X-ray CT test is useful to gain insight into the influence of material's structure and moisture dynamics on its fungal susceptibility. It allows us to gather localized 
information on fungal activity during the entire degradation process, without disrupting it.

\section{Acknowledgements}

The authors gratefully acknowledge the financial support of the Research Foundation Flanders (FWO SB grant $1 \mathrm{~S} 53417 \mathrm{~N})$.

\section{ORCID}

Liselotte De Ligne: http://orcid.org/0000-0002-2202-42208

Jan Van den Bulcke: http://orcid.org/0000-0003-2939-5408

Jan M. Baetens: http://orcid.org/0000-0003-4084-9992

Bernard De Baets: http://orcid.org/0000-0002-3876-620X

Joris Van Acker: https://orcid.org/0000-0002-8961-0176

References

Adan, O.C. and Samson, R.A. (2011). Fundamentals of Mold Growth in Indoor Environments and Strategies for Healthy Living. Wageningen, Wageningen Academic Publishers.

Bergsten, U., Lindeberg, J., Rindby, A. and Evans, R. (2001). Batch measurements of wood density on intact or prepared drill cores using x-ray microdensitometry. Wood Science and Technology, 35, 435-452. doi: $10.1007 / \mathrm{s} 002260100106$

Bertron, A. (2014). Understanding interactions between cementitious materials and microorganisms: a key to sustainable and safe concrete structures in various contexts. Materials and Structures, 47, 1787-1806. doi: $10.1617 /$ s $11527-014-0433-1$

Candelier, K., Thevenon, M. wood thermal treatment a doi: $10.1007 / \mathrm{s} 13595-016-0$

CEN (1996). EN 113: Woor wood destroying basidionycetes

CEN (2016). EN 350: Durability of wo
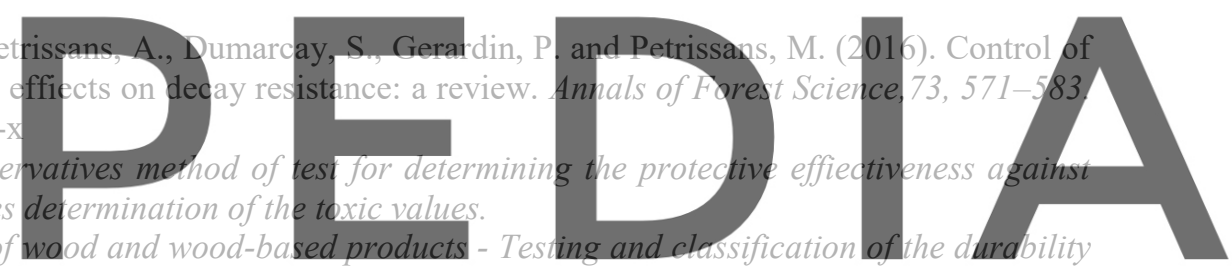
to biological agents of wood and wood-based materials.

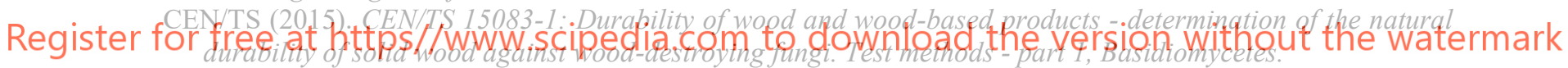

Chen, S.G., Liu, X.H., Fang, L.M. and Wellwood, R. (2010). Digital X-ray analysis of density distribution characteristics of wood-based panels. Wood Science and Technology, 44, 85-93. doi: 10.1007/s00226-0090256-3

De Ligne, L., Van den Bulcke, J., De Muynck, A., Baetens, J., De Baets, B., Van Hoorebeke, L. and Van Acker, J. (2019). Exploring the use of X-ray micro CT as a tool for the monitoring of moisture production and mass loss during lab-based fungal degradation testing. In Proceedings of the $50^{\text {th }}$ Annual Meeting of the International Research Group on Wood Protection, Quebec city, Canada, IRG/WP 19-20654.

Dedesko, S. and Siegel, J.A. (2015). Moisture parameters and fungal communities associated with gypsum drywall in buildings. Microbiome, 3, 1. doi: 10.1186/s40168-015-0137-y

Freyburger, C., Longuetaud, F., Mothe, F., Constant, T. and Leban, J.M. (2009). Measuring wood density by means of X-ray computer tomography. Annals of Forest Science, 66, 804. doi: 10.1051/forest/2009071

Ganotopoulou, E. (2014). Biodegradable materials: A research and design handbook; enhancing the use of biodegradable materials on building's envelopes in the Netherlands(in English), PhD thesis, Delft University, Delft, The Netherlands.

Goodell, B., Nicholas, D.D. and Schultz, T.P. (2003). Wood deterioration and preservation: Advances in our changing world. Oxford, Oxford University Press.

Herczeg, M., McKinnon, D., Milios, L., Bakas, I., Klaassens, E., Svatikova, K. and Widerberg, O. (2014). Resource efficiency in the building sector, Final report prepared for the European Commission, ECORYS and Copenhagen Resource Institute, Rotterdam, the Netherlands.

Hoang, C.P., Kinney, K.A., Corsi, R.L. and Szaniszlo, P.J. (2010). Resistance of green building materials to fungal 
growth. International Biodeterioration \& Biodegradation, 64, 104-113. doi: 10.1016/j.ibiod.2009.11.001

Homan, W.J. and Jorissen, A.J. (2004). Wood modification developments. Heron, 49, 360-369.

Jebrane, M., Pockrandt, M. and Terziev, N. (2014). Natural durability of selected larch and Scots pine heartwoods in laboratory and field tests. International Biodeterioration \& Biodegradation, 91, 88-96. doi: 10.1016/j.ibiod.2014.03.018

Jones D. and Brischke C. (2017). Performance of Bio-Based Building Materials. Duxford, Woodhead Publishing: Elsevier.

Kamdem, D., Pizzi, A. and Jermannaud, A. (2002). Durability of heat-treated wood. Holz als Roh-und Werkstoff, 60, 1-6. doi: 10.1007/s00107-001-0261-1

Kutnik, M. (2013). Focus on the european standardization - towards a revision of the en 350 natural durability standard: a diffierent approach to the inherent resistance and performance of wood and wood-based materials. In Proceedings of the $44^{\text {th }}$ Annual Meeting of the International Research Group on Wood Protection, Stockholm, Sweden, IRG/WP 13-10811.

Kutnik, M., Suttie, E. and Brischke, C. (2014). European standards on durability and performance of wood and wood-based products-trends and challenges. Wood Material Science and Engineering, 9, 122-133. doi: $10.1080 / 17480272.2014 .894574$

Li, W. (2016). Experimental investigation of the relation between structure and moisture behaviour of wood-based panels (in English), $\mathrm{PhD}$ Thesis, Ghent University, Ghent, Belgium.

Mai, C., Verma, P., YanJun, X., Dyckmans, J. and Militz, H. (2010). Protection mechanisms of modified wood against decay by white and brown rot fungi. In Proceedings of the $41^{\text {st }}$ Annual Meeting of the International Research Group on Wood Protection, Biarritz, France, IRG/WP 10-10713.

Mazela, B., Zakrzewski, R., Grze'skowiak, W., Cofta, G. and Bartkowiak, M. (2004). Resistance of thermally modified wood to basidiomycetes. Electronic Journal of Polish Agricultural Universities, 7, 1505-0297.

Melchert, L. (2007). The Dutch sustainable building policy: A model for developing countries? Building and Environment, 42, 893-901. doi: 10.1016/j.buildenv.2005.10.007

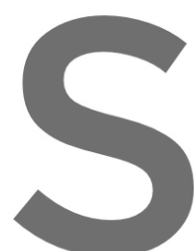

Ormondroyd, G., Spear, Proceedings of the 10.1680/coma.14.00072

Ringman, R., Pilgffiard, modified wood: a review

Sathre, R. and O'Connor
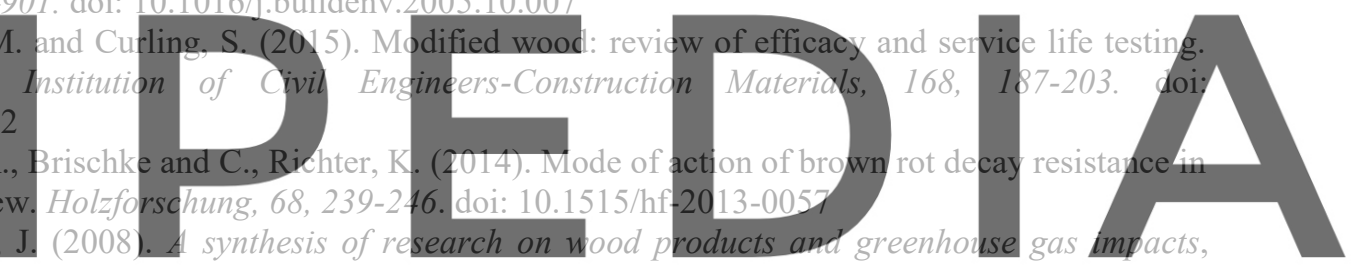

Technical report TR-19R, FPInnovations, Vancouver, Canada.

Register formidt, O. 2006. Wogd and Tree Fungi: Biology, Damage, Protectipn and Use Berlin, Springer-Verlag. materials and sampling and analysis methods. Building and Environment, 80, 136-149. doi: 10.1016/j.buildenv.2014.05.030 